\title{
Zum Problem der Waldhufen-Siedlungen am Beispiel des Schweizer Juras
}

\section{Bemerkungen zur Typologie der Waldhufensiedlung}

Überlegungen zur Typologie der Waldhufensiedlung werden sich in jedem Fall hauptsächlich mit der grundlegenden Arbeit von R. KRÜGER (1967) auseinandersetzen müssen. Hier wurde erstmalig versucht, in die verwirrende Vielfalt der Begriffe, die seit der ersten Beschäftigung mit diesen Siedlungsformen durch V.JACOBI (1845) aufgebracht worden sind, eine ordnende Systematik zu bringen, die als Diskussionsgrundlage außerordentlich wertvoll ist. Vor einer Auseinandersetzung mit den Thesen KRÜGERS muß in aller Kürze nochmals an einige formale Grundbegriffe erinnert werden, die dem Fachkundigen freilich selbstverständlich sind. Unter einer Waldhufensiedlung versteht man im heutigen geographischen Sprachgebrauch eine Rodungssiedlung im ehemaligen Waldland, die durch eine Kombination von Orts- und Flurform, nämlich eine reihenförmige, ein- oder zweizeilige Ortsanlage entlang einer Straße oder einem Bach und eine aus hofanschließenden, senkrecht zur Siedlungsachse verlaufenden Streifenparzellen bestehende Flur charakterisiert ist.

Eine Waldhufenflur besteht aus mehreren "gereihten Streifen in Besitzeinheit mit Hofanschluß» (G. SCHWARZ, 1966, S. 206 ff.). Man kann die Waldhufenfluren auch als Verband von in ihrer Länge (und Breite) variierbaren Streifenparzellen in Einödlage mit Hofanschluß bezeichnen, als «Breitstreifeneinödparzellen mit Hofanschluß» (vgl. C. LIENAU, 1967, S. 121). Diese Definition ist als Rahmendefinition gedacht, unter die sämtliche «Hufensiedlungen» untergeordnet werden können. Dazu gehören also «Waldhufensiedlungen» als Rodungssiedlungen in ehemaligem Waldland, "Hagenhufensiedlungen» als landschaftlich begrenzte, meist einzeilige Sonderformen sowie Marsch- und Moorhufensiedlungen als Kolonisationsformen im Marsch- und Moorgebiet. In der Forschung wurde früh schon die Problematik dieser Bezeichnungen, speziell der des Waldhufendorfes erkannt, da dieses Wort in seinem heutigen Bedeutungsinhalt etwas anderes meint als im Mittelalter das Wort "Waldhufe» oder "Hufe», das ja in allen vorhin genannten Typen enthalten ist.

Außerdem bemerkte man, daß man mit dem Begriff "Waldhufendorf» nicht der ganzen Vielfalt von
Siedlungen mit physiognomisch ähnlicher Struktur gerecht wurde.

Zur Bedeutungsentwicklung des Begriffes «Hufe» kann man sich auf den vorzüglichen kurzen Abriß von R. KRÜGER stützen (S.17 ff.). Es geht schon aus den dort aufgeführten Beispielen (vgl. auch R. KöTZSCHKE, 1953) hervor, wie schwierig es ist, den Begriff «Hufe» in seinem Bedeutungsinhalt für die jeweilige Zeit genau zu definieren. Die «Hufe» bedeutete einmal eine bäuerliche Siedelstelle einschließlich der dazugehörigen Nutzungsrechte an der allgemeinen Dorfmark (F. LÜTGE, 1963, S. 74 und H. CONRAD, 1962, Bd. I, S. 85).

Dann war die «Hufe» ein "Stück Land von gewisser Größe» (F. KLUGE, 1963, S. 318). Der althochdeutsche Begriff «huoba», mittelhochdeutsch «hoeve» entsprach dem lateinischen Wort "mansus» und bezeichnete wie dieses die Wohn- und Wirtschaftsstelle eines Bauern im Sinne einer bäuerlichen Besitzeinheit. (R. KRÜGER, 1967, S.17, zit. W. RÖPKE, 1924, S. 35, Anm. 57). Obwohl "Hufe» und "mansus» ursprünglich nicht ganz synonym gewesen sein mögen, bekamen sie doch mit der Zeit die gleiche Bedeutung, so daß ein "mansus» eine "Hufe Lands auf dem Felde» war (R. KÖTZSCHKE, 1938, S. 246).

Nach einigen unklaren mittelalterlichen Erwähnungen aus dem 11. und 13. Jh. von "hobis silve» und "walt hube» (bei R. KRÜGER, S. 18 und 19) tauchen später festumrissene Ackermaße auf, die als «mansus franconicus» bzw. als "mansus magnus» bezeichnet werden. Bei diesen Maßen handelt es sich um die sogenannte "fränkische Hufe» (23,2 ha); daneben ist die "flämische Hufe» (16,8 ha) häufig anzutreffen. Beides sind festumrissene und speziell für die deutsche Ostkolonisation höchst wichtige Flächenmaße, die jedoch niemals als "Waldhufe» bezeichnet werden.

Man kann in allen Punkten KRÜGERS Schlußfolgerungen (S.20) zustimmen, wenn er ausführt:

1) Besitzeinheiten, die auf gerodetem Waldland geschaffen wurden, wurden ohne Ansehen der Form als "Hufe», möglicherweise als «hobae silve» oder «walt hube» bezeichnet.

Dr. Werner Kreisel, Geogr. Inst. der Rhein.-Westf. Techn. Hochschule, Templergraben 55, D-51 Aachen 
2) Später bezeichnete "mansus magnus» oder "mansus franconicus» (nicht "Hufe» oder "Waldhufe») die «fränkische Hufe».

3) Ein Bedeutungsinhalt, der im heutigen formaltypologischen Sinne eine bestimmte Siedelform mit einstreifigen, hofanschließenden Besitzeinheiten meint, ist aus diesen mittelalterlichen Quellen nicht abzulesen (S. 20).

«Hufe» im historischen Sinne, also in der Bedeutung, wie das Wort im Mittelalter gebraucht wurde, hat also nichts mit dem geographischen Begriff zu tun, der unter einer Waldhufenflur eine Aneinanderreihung von gereihten Streifen in Besitzeinheit mit Hofanschluß versteht. Der geographische Begriff "Waldhufe» stellt sich so als eine "gelehrte Bildung», eine künstliche Neuschöpfung heraus, die den Hufenbegriff - methodisch seinerzeit nicht ganz glücklich - nur auf die Rodungsreihensiedlungen mit hofanschließender Breitstreifeneinödflur anwendete. Bei den derartig geformten Reihendörfern war zugegebenermaßen die fränkische Hufe zum Teil gleichzeitig das Flächenmaß jedes hofanschlieBenden Streifens. Doch ist dieses $\mathrm{Ma}$ eben nicht nur bei diesen Rodungs-Reihensiedlungen vorhanden, sondern z. B. auch bei den Plangewannfluren der ostdeutschen Kolonisation, die man ja auch "Hufengewannflur» nannte, weil die in den drei Plangewannen verteilten Besitze insgesamt den Flächeninhalt einer fränkischen Hufe haben (A. KRENZLIN, 1953, S. 25 ff.).

Nach KRÜGER lassen sich die «Breitstreifenreihendörfer mit oder ohne Zusatzstreifenflur» (BRZ) formal gliedern in die Normform (Hochform), in die Vorausformen und formverwandte Hochformen (Westdeutschland) sowie die Auflösungsformen. Ihnen ist gestaltmäßig verwandt eine Gruppe von Siedelformen, die «formal ähnliche Siedelformen» benannt wurde (S.76). Bedenklich ist es von vornherein, bei einer formalen Typisierung Begriffe wie "Hochform» oder Vorausform» zu verwenden. Selbst wenn es sich herausstellen sollte, daß die "Vorausformen" tatsächlich eine Entwicklungsstufe zu den Hochformen sein sollten, so ist es doch methodisch ein ungangbarer Weg, wenn man dies in ein formales Typenschema einbauen wollte. "Hochform» und "Vorausformen" sind eben keine formaltypologischen Begriffe, und wieder wird hierin die Vermengung von genetischen und formalen Termini deutlich, wie sie schon H.ZSCHOCKE (1970, S. 58) festgestellt hat.

KRÜGER (S. 70 f.) bindet den Terminus «Waldhufendorf» «im streng wissenschaftlichen Sinn - historisch... an die Benennung der Siedelformen, die unter deutschem Recht während der mittelalterlichen Rodekolonisation der mittel- und ostdeutschen sowie karpatischen Gebirge entstanden sind». Aufgrund der unberechtigten historischen Beziehung zur fränkischen Hufe wird vorgeschlagen, «als Waldhufendorf nur die Siedelformen zu bezeichnen, bei denen sich harmonisch eine Doppelgehöftreihe und hofanschließende Breitstreifen von der Größe der uniform angewandten fränkischen Hufe (24,2 ha

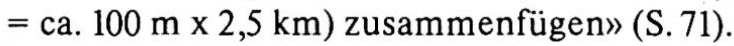

Dies ist zwar ein Lösungsversuch, doch wird hierdurch keine Klarheit geschaffen, sondern verschleiert, daß physiognomisch diese Flurform mit verschiedenen Ortsformen zu kombinieren ist.

Der geographische Begriff «Waldhufensiedlung» hat jedoch keine immanente Herleitung vom historischen. Die Festlegung des Begriffs "Waldhufendorf» auf die damit gemeinte Siedlungsform ist eine Konstruktion, eine Übertragung eines historisch schillernden Begriffes in einen physiognomischen. Dann ist es auch nicht zulässig, die Waldhufensiedlung mit einer bestimmten Maßeinheit (fränkische Hufe) zu verknüpfen. Es ist nicht angängig, den im «formaltypologischen» Sinne angewendeten Begriff "Waldhufe» im nachhinein historisch zu belasten (vgl. H. ZSCHOCKE, 1970, S. 57 f.). Formaltypologisch entspricht "Waldhufensiedlung» der «Reihensiedlung mit hofanschließender Breitstreifen-Einödflur», als Sonderfall der in ehemaliges Waldland gerodeten Siedlungen. Für die Formaltypologie gilt es zunächst, diese Siedlungsformen physiognomisch zu beschreiben und unbeeinflußt von der Genese Typen zu bilden. Dann erst muß als zweiter Schritt die Genese betrachtet und beides schließlich superponiert werden, aber nicht von vornherein dürfen beide Gesichtspunkte vermengt werden. Um Definitionsverwirrungen zu vermeiden, würde man den Begriff "Waldhufen-» besser ganz aufgeben, als ihn historisch zu belasten. Statt «Waldhufe-» sollte man dann lieber vom «Normalfall der Rodungs-Reihensiedlung mit Breitstreifen-Einödparzellen und Hofanschluß» reden und damit das bisherige "Waldhufen- 
dorf» meinen. Statt dieser komplizierten Formulierung könnte man einfach den Begriff «Waldhufensiedlung» beibehalten. Man kann dann darangehen, ähnliche Formen formaltypologisch zu erfassen, im Bewußtsein, daß das System der gereihten Streifen in Besitzeinheit und Hofanschluß nicht auf die Ortsform des Reihendorfes beschränkt ist, sondern auch bei linear gerichteten kleineren Gruppensiedlungen (Weilern) und bei gereihten Einzelhöfen auftritt.

Es ist selbstverständlich, daß ein Breitstreifenreihendorf eine andere Entstehung haben mag als eine Einzelhofreihe mit Waldhufenflur. Das hat K.A. HABBE (1966, S. 52) noch einmal für das Hofsiedlungsgebiet des mittleren Schwarzwaldes betont und genetisch keine Verbindung mit den Waldhufendörfern des Odenwaldes herstellen können. Dasselbe ergaben auch eigene Untersuchungen im Schweizer Faltenjura, wo ebenfalls eine verschiedene Art und Zeit der Anlage verschiedenartiger Siedlungen festgestellt werden konnte (W.KREISEL, 1972). Hier kommt es aber zunächst nicht auf die genetischen Unterschiede, sondern auf die physiognomische Ähnlichkeit an. Auch H. J. NITZ (1962) sucht keine genetische Verwandtschaft zwischen Waldhufendörfern und Zinken, sondern nur eine physiognomische.

Vom klassischen Waldhufendorf unterscheiden sich solche Anlagen, die mit den Ortsformen des Weilers oder der Einzelhofreihe verknüpft sind, nicht grundsätzlich in der Struktur der gereihten Streifen, sondern nur durch den verschiedenen Abstand der Höfe untereinander, somit der Breite und der Größe der Streifen. Es handelt sich hierbei nur um zwei Extremfälle desselben Prinzips, als dessen charakteristische und häufigste Form das Waldhufendorf zu gelten hat.

Durch die physiognomisch gemeinte Bezeichnung "Waldhufe» erhält man die Flurstruktur der Breitstreifeneinödparzellen einerseits - rein beschreibend als "Waldhufenflur» bezeichnet, die einzelne Breitstreifeneinödparzelle als "Waldhufe» - und die Ortsform des Reihendorfes, des Reihenweilers oder der gereihten Einzelhöfe andererseits. Durch jeweilige Kombination von beidem ergibt sich dann die Möglichkeit einer ersten groben Typisierung, als Reihendorf mit Waldhufenflur, Reihenweiler mit Waldhufenflur oder gereihte Einzelhöfe mit Waldhufenflur.

Der Oberbegriff, der ja alle Normvarianten, also auch gereihte Streifen in Besitzeinheit mit Hofanschluß in Verbindung mit anderen Ortsformen als dem Dorf umfassen soll, kann daher nicht Breitstreifenreihendorf lauten. Reihenweiler und gereihte Einzelhöfe sind eben keine Dörfer. In der Zusammenstellung von KRÜGER scheint die Verschiedenartigkeit des Ortsbildes bei den einzelnen Varianten zu wenig berücksichtigt. Die Trennung von Orts- und Flurform bei diesen Siedlungsanlagen, die schon bei H. J.NITZ (1962, S. 80 ff.) empfohlen war, wird hier zu sehr vernachlässigt. Man sollte also "Breitstreifenreihendorf» durch "Breitstreifenreihensiedlung» ersetzen. Wie schon bei H. J. NITZ $(1962$, S. 50) und H.ZSCHOCKE $(1963$, S.1) wird auch an dieser Stelle für die Beibehaltung des Suffixes «Siedlung» anstatt «Dorf» plädiert, da "Siedlung» in jedem Fall der allgemeinere Terminus ist als «Dorf».

Diese beiden Sondertypen sind bei KRÜGER unter d3) und d2) angedeutet (S.75, s. auch S. 67). Statt "Weilerwaldhufendorf» (H. GRAUL, 1942, S. 81) wird der Terminus «Zwei Gehöftreihen mit hofanschlieBenden, in sich zersplitterten Breitstreifen $\left(2 \mathrm{GR}_{\mathrm{e}} /\right.$ B : $x$ )" gewählt. Im geographischen Sprachgebrauch gilt der Weiler rein formaltypologisch als eine kleine Gruppensiedlung von 10-20 Gehöften. Von hier aus liegt also bei Außerachtlassen der früher üblichen historischen Belastung des Wortes kein Anlaß vor, diesen Begriff zu meiden. "Weilerwaldhufendorf» ist freilich nicht besser als KRÜGERS Formulierung, da Weiler und Dorf sich gegenseitig ausschließen. «Reihenweiler mit hofanschließender Breitstreifeneinödflur» wäre hier vorzuziehen.

Die Existenz von gereihten Streifen bei Einzelhöfen erwähnt KRÜGER nur ganz am Rande. Er nennt lediglich unter $\mathrm{d} 2$ ) das "(Doppel)reihendorf übergehend in Streusiedlung mit hofanschließenden Breitstreifen mit teilweiser Auflösung der Flur in Blöcke». Diesem Typ gibt er die Formel (2) $R_{e}+$ $\mathrm{S} b / \mathrm{B}+\mathrm{BI}$. Es zeigt sich bei KRÜGERs Schema ein Mangel von Begriffen, die abstrakt und umfassend genug sind, um die Gesamtheit der konkreten Sachverhalte klar zum Ausdruck kommen zu lassen. Dies wird klar im Außerachtlassen der Hauptvariationsmöglichkeit in "Dorf», Weiler» und «Einzelhöfe», wie in der von vornherein festgelegten Einschränkung auf ganz bestimmte Ausprägungsformen. Dies kann in einem formalen Typenschema nicht in Betracht kommen. 
Wenn darüber hinaus Definitionen wie die genannten in Fremdsprachen übersetzt werden sollen, die nicht wie das Deutsche die Möglichkeit haben, mehrere verschiedene Wörter durch Aneinanderreihen zu einem neuen zu verschmelzen, treten einige Schwierigkeiten auf. So ist im Französischen oftmals versucht worden, den vergleichsweise einfachen Begriff der «Breitstreifenflur» aus dem Deutschen zu übersetzen. Dabei bedarf der im Deutschen in einem Wort ausdrückbare Terminus im Französischen eines ganzen Satzes (vgl. H. UHLIG, 1967, S. F32f.). Noch umständlicher wäre eine Übersetzung von «Rodungs-Reihensiedlung mit hofanschließender Breitstreifeneinödflur».

Daher ist man nach mehreren Übersetzungsversuchen (so A.DEMANGEON, 1946, S. 189 «village allongé», R. LEBEAU, 1960, S. 21 «village linéaire») dazu übergegangen, den deutschen Terminus «Waldhufensiedlung» und "Waldhufendorf» im genannten Sinn als Oberbegriff zu akzeptieren und dann die verschiedenen Ausprägungsformen zu beschreiben (vgl. H. UHLIG, 1967, S. F 33, R. LEBEAU, 1969, S. 23, 43). Im Grunde genommen ist es nicht einzusehen, da $B$ man den Begriff «Waldhufe-» auch im Deutschen, so wie er sich eingebürgert hat, mit der vorhin genannten Bedeutung weiterverwenden sollte.

Dadurch, daß die Maßeinheit der fränkischen Hufe zugrunde gelegt wird, entfällt für KRÜGER die Möglichkeit, andere Gebiete, in denen Breitstreifenreihensiedlungen mit Hofanschluß, aber anderem Flächenmaß auftreten, in Betracht zu ziehen. Die in Frankreich festgestellten ähnlichen Siedlungsreihen, wie sie in der Champagne, der Normandie, der Picardie vorkommen (vgl. A. DEMANGEON, 1946, S. $188 \mathrm{ff}$.), werden ebensowenig berücksichtigt, wie die des Schweizer und Französischen Jura (W. KREISEL, 1972). Ähnliche Siedlungen gleicher Formalstruktur in Südbrasilien (s. WAIBEL, 1955), in Canada (F. BARTZ, 1955), in Bolivien (w. SCHOOP, 1971), in Japan (M. SCHWIND, 1943), in Israel (W. RICHTER, 1969), in Ghana (W. MANSHARD, 1961) und in vielen weiteren Gebieten werden übergangen. Selbst wenn diese nicht als "Waldhufendörfer» in KRÜGERs Sinn verstanden werden, weil die Hufenbemessung eine andere war als die der fränkischen Hufe, müßte man in irgendeiner Form auf sie als ähnliche Siedlungsformen hinweisen. Hier zeigt sich ein weiterer Man- gel dieses Schemas darin, daß sie dort eben nicht einzuordnen sind.

Das Typenschema von KRÜGER beinhaltet folgende Begriffe: BRZ (Breitstreifensiedlung mit oder ohne Zusatzstreifen) sind durch folgende formale Kriterien bestimmt:

a) R - Reihe, lockere Reihung der Gehöfte Abgrenzung gegen formal ähnliche Dorfformen: 1. Z - Zeile, Zusammenrücken der Gehöfte auf weniger als $40 \mathrm{~m}$ Hofabstand (geschlossene Bebauung)

2. Einzelhof, Einödhof, Auseinanderrücken der Gehöfte auf mehr als 150 m Hofabstand (Aufgabe eines planmäßig gewollten Dorfgrundrisses) Modifikationen der lockeren Reihe sind gegeben mit:

1. KR - Kurzreihe, Längserstreckung der Reihe verkürzt (entstanden durch Zusammenrücken der Höfe auf unter $100 \mathrm{~m}$ Abstand, und damit meist verbunden durch Verringerung der Gehöftzahl auf weniger als 25)

2. GR - Gehöftgruppenreihe, Bildung von gereihten Gehöftgruppen (je 2-4 Höfe)

3. 2R - Doppelreihe,

$\mathrm{R}$ - Einzelreihe

b) B - Breitstreifen, gereiht und hofanschließend (mit einer Breite von 40-150 m und einer Länge von über $1000 \mathrm{~m}$ )

Modifikationen der Breitstreifen sind gegeben mit:

1. Bk. - kurze Breitstreifen, unter $1000 \mathrm{~m}$ lang

2. Br. - Breitstreifen, die fächerförmig von der Gehöftreihe oder von Abschnitten der Gehöftreihe nach außen streben

Z - Zusatzstreifen, gereiht und nicht hofanschließend, meist geschlossen in Gewannen liegend

c) Die Kopplung von Ortsgrundriß und Breitstreifenflur wird in der Art des Hofanschlusses signifikant:

1. $R_{e}$ - am Sreifenende (Kopfende des Streifens)

2. $R_{m}$ - in Streifenmitte

3. $R_{s}-z u$ einer Seite der Streifen hin

4. $R_{b}$ - an beliebig wechselnder Stelle innerhalb der Breitstreifenflur

Abgesehen von den in das Typenschema nicht gehörenden genetischen Begriffen ist dieses schon im 
Deutschen so umständlich, die Abkürzungen für die einzelnen Definitionsbestandteile so unübersichtlich und verwirrend, daß es vollends unbrauchbar wird, wenn man versucht, es etwa in einer Fremdsprache auszudrücken. Besonders die Abkürzung durch Buchstaben, den Anfangsbuchstaben der deutschen Termini, ist völlig auf die deutsche Sprache festgelgt.

Diesen Schwierigkeiten könnte man zu entgehen versuchen, wenn man statt der Buchstabenabkürzungen Zahlen oder Ziffern nähme und praktisch im Sinne der Dezimalmethode einen formalen Typ durch eine bestimmte Ziffernkombination ausdrückte. Als Oberbegriff für alle Siedlungen dieser Art muß u. E. «aus Rodung hervorgegangene Reihensiedlung mit hofanschließenden Breitstreifeneinödparzellen» (Marschhufen- und Moorhufensiedlungen fallen dabei weg, werden jedoch als ähnliche Siedlungsformen anerkannt) oder in unserem beschreibenden Sinne einfach «Waldhufensiedlung» gewählt werden.

Die Differenzierung könnte dann etwa so erfolgen, wie KRÜGER sie vornimmt, doch müssen auf jeden Fall die wesentlichen Differenzierungen der Ortsform ausgedrückt werden: Reihendorf, Reihenweiler, gereihte Einzelhöfe. Die Grenzwerte zwischen den drei Typen müßte man nach der gängigen Definition festlegen, ohne jedoch in ein zu starres Schema zu verfallen, das dem konkreten Sachverhalt nicht mehr gerecht würde.

Versuchsweise könnte man die drei Typen mit Ziffern benennen. Das Reihendorf mit 1., den Reihenweiler mit 2., die gereihten Einzelhöfe mit 3., Sonderformen wie das Radialwaldhufendorf mit 4. Diese Ziffer bekäme die erste Stelle in der endgültigen $\mathrm{Zahl}$, die den festgestellten Formaltyp bezeichnen würde.

Hier sollen nicht sämtliche Kriterien wiederholt werden, nach denen sich Waldhufensiedlungen unterscheiden können, sondern nur ein paar wesentliche Variationsmöglichkeiten aufgezeigt werden. Ein solches Unterscheidungskriterium wäre etwa die Anzahl der Häuserzeilen. Bei Einzeiligkeit setzte man an die zweite Stelle der Formel die Ziffer 1., bei Zweizeiligkeit die Ziffer 2. Weiterhin könnte man in kurze und lange Hufen differenzieren, bei kurzen wäre an die dritte Stelle der Formel eine 1. zu setzen, bei langen eine 2. Damit wären schon die wesentlichen formaltypologischen Kriterien erfaßt.
Man könnte dann darangehen, Zeit und Umstände der Siedlungsanlagen in der gleichen Formel darzustellen: Für den europäischen Bereich käme eine Einteilung etwa in hochmittelalterliche Gründungen und nachmittelalterliche Gründungen in Frage, wieder ausdrückbar durch die Ziffern 1. und 2. an der vierten Stelle der Formel. - Für außereuropäische Gebiete wird diese Unterscheidung nicht ausreichen, man müßte sich überlegen, ob man hier nicht das Jahrhundert oder ein noch genaueres Datum der Gründung angeben müßte, was freilich einen Bruch im System darstellen würde. Für das theoretische Durchspielen des Schemas mag dies jedoch genügen.

$\mathrm{Da}$ alle Waldhufensiedlungen ein gewisses $\mathrm{Ma} ß$ an Planung voraussetzen, könnte man - theoretisch als die zwei Extreme eine intensiv gelenkte Siedlungsanlage (durch Grundherren, Landesherren, staatliche Organe, Organisationen usw.) auf der einen und eine durch Absprache der Siedler selbst getroffene Vereinbarung auf der anderen Seite annehmen und an die fünfte Stelle der sich ergebenden Endzahl wieder die Ziffern 1. und 2. setzen. Weitere Kriterien, wie Art der landwirtschaftlichen Nutzung, System der Erbfolge usw. könnte man zwanglos anschließen. Um vielleicht noch deutlicher zu machen, daß Zeit der Gründung und Art der Siedlungsanlage keine formaltypologischen Kriterien sind, könnte man sie gegen diese dadurch absetzen, da $\beta$ man in diesem Fall statt der Ziffern Buchstaben einsetzt, also a. und b. Sollte sich zeigen, daß diese Zifferngliederung immer noch zu kompliziert ist, kann man die Ziffern dadurch ersetzen, daß man für jedes Kriterium ein anderes Kennzeichen nimmt, also z. B. für die Ortsformen A. B. C., für Einzeiligkeit und Zweizeiligkeit 1. und 2., für Länge der Hufen (kurz und lang) I. und II., für mittelalterliche bzw. nachmittelalterliche Entstehung a. und b., für starke bzw. schwache Intensität der Planung $\alpha$ und $\beta$ usw.

Es ergäben sich so drei verschiedene Möglichkeiten einer Schematisierung:

Ein zweizeiliges Waldhufendorf mit langen Hufen, das durch grundherrschaftliche Initiative im Mittelalter angelegt worden ist, wäre dann:

entweder 1.2.2.1.1.

oder 1.2.2.a.a.

oder A.2.II.a.a. 
Schema 1 Schema 2 Schema 3

\begin{tabular}{lllll}
\hline 1. Kriterium: Ortsform: & Reihendorf & 1 & 1 & $\mathrm{~A}$ \\
\cline { 2 - 5 } (1. Stelle der Endformel) & Reihenweiler & 2 & 2 & $\mathrm{~B}$ \\
\cline { 2 - 5 } & Einzelhofreihe & 3 & 3 & $\mathrm{C}$ \\
\hline 2. Kriterium: Häuserzeilen: & eine & 1 & 1 & 1 \\
\cline { 2 - 5 } (2. Stelle der Endformel) & zwei & 2 & 2 & 2 \\
\hline 3. Kriterium: Hufenlänge: & kurz & 1 & 1 & $\mathrm{I}$ \\
\cline { 2 - 5 } (3. Stelle der Endformel) & lang & 2 & 2 & $\mathrm{II}$ \\
\hline 4. Kriterium: Zeit der Siedlungsanlage: & Mittelalter & 1 & $\mathrm{a}$ & $\mathrm{a}$ \\
\cline { 2 - 5 } (4. Stelle der Endformel) & Nachmittelalter & 2 & $\mathrm{~b}$ & $\mathrm{~b}$ \\
\hline 5. Kriterium: Intensität der Planung: & $\begin{array}{l}\text { durch planende Initiative von } \\
\text { srundherren oder Organi- }\end{array}$ & 1 & $\mathrm{a}$ & $\alpha$ \\
\cline { 2 - 5 } & $\begin{array}{l}\text { dutionen angelegt: } \\
\text { der Siedler angelegt: }\end{array}$ & 2 & $\mathrm{~b}$ & $\beta$ \\
\hline
\end{tabular}

Eine einzeilige Einzelhofreihe mit langen Hufen und einer mehr individuellen, nachmittelalterlichen Entstehung wäre dann:

$\begin{array}{ll}\text { entweder } & 3.1 .2 .2 .2 . \\ \text { oder } & 3.1 .2 . b . b . \\ \text { oder } & \text { C.1.II.b. } \beta .\end{array}$

Auf diese Weise scheint eine Typisierung etwas übersichtlicher zu sein als nach dem KRÜGERschen Schema. Das Vorgeschlagene läßt sich beliebig ergänzen und differenzieren.

Zusammenfassend läßt sich u. E. feststellen: es geht nicht an, aufgrund einer etymologischen Herleitung aus einem historischen Begriff einen neu geschaffenen geographischen Terminus physiognomischen Inhalts zu beschränken. "Waldhufe-» kann daher nicht nur auf das "Waldhufendorf» der deutschen
Ostkolonisation mit dem üblichen Parzellenmaß der fränkischen Hufe angewendet werden. «Waldhufensiedlung» ist vielmehr im physiognomischen Sinne ein Oberbegriff über sämtliche «aus Rodung hervorgegangenen Reihensiedlungen mit hofanschlieBenden Breitstreifeneinödparzellen» und entspricht diesem letzten Begriff als Synonym. Daher ist auch im streng wissenschaftlichen Sinn und der Einfachheit halber neben der Form des "Waldhufendorfes» die Bezeichnung "Waldhufenweiler» und «Einzelhofreihe mit Waldhufenflur» berechtigt. Alle diese Sonderformen sind Ausprägungen desselben Prinzips, sie stellen mehr oder weniger eng gedrängte Rodungsreihensiedlungen mit hofanschließenden Breitstreifeneinödparzellen dar, die als mehr oder weniger intensiv geplante Kolonisationsformen zu verschiedenen Zeiten entstanden sein können. 


\section{Waldhufensiedlungen im Jura}

S. DAVEAU (1959), H. GUTERSOHN (1958) und R. LEBEAU (1960) hatten im Jura bereits waldhufenähnliche Siedlungen festgestellt, sie beschrieben und umschrieben als «villages allongés» oder «villages linéaires» («Reihensiedlungen») und ihre Flur als «fischgrätenartig» strukturiert bezeichnet («un finage en arête de poisson»). LEBEAU glaubte die Anlage dieser Siedlungen ins 16.-17. Jh. setzen zu müssen, eine Ansicht, die freilich revidiert werden muß, ebenso wie diese Waldhufensiedlungen genauer zu differenzieren sind:

Bei eingehender Beschäftigung ergibt sich, daß hauptsächlich zwei Spielarten auftreten, nämlich das Waldhufendorf und die Einzelhofreihe mit Waldhufenflur. Außerdem konzentrieren sich diese Siedlungen in bestimmten Bereichen des Hochjura:

a) Im Gebiet zwischen Neuchâtel und Pontarlier die Waldhufendörfer Les Verrières-de-Neuchâtel, Les Bayards, Les Fourgs und L'Auberson sowie die Einzelhofreihen der Täler von La Brévine und Les Ponts-de-Martel

b) Im Joux-Tal die gereihten Einzelhöfe der Gemeinden Le Chénit und Bois-d'Amont

c) Im Grandvaux die gereihten Einzelhöfe der Gemeinden Grande-Rivière, Rivière-Devant, St.Pierre, La Chaumusse, Fort-de-Plasne und Lac-des-Rouges-Truites

Das Waldhufendorf soll nun am Beispiel von Les Verrières-de-Neuchâtel und die Einzelhofreihe am Beispiel des Tales von La Brévine besprochen werden.

\section{Les Verrières-de-Neuchâtel}

Les Verrières-de-Neuchâtel liegt in der durch Kreideund Malmkalke aufgebauten, glazial überformten Synklinale von Les Verrières, an der wichtigen Durchgangsverbindung von Neuchâtel nach Pontarlier. Durch die Funktion des Ortes als Grenzbahnhof und als Zollstation kam es zu einer starken Überformung durch die hierdurch bedingten Gebäude und Anlagen sowie durch die zahlreichen Wohnhäuser für die Beschäftigten des tertiären Sektors.
Die Flur ist nach der Terminologie von R. ZSCHOCKE eine Streifen-Planflur, die auf eine Flurbereinigung in den Jahren 1946-1954 zurückgeht. Dennoch ergeben sich bereits hieraus einige Anhaltspunkte für das frühere Aussehen der Flur:

1) Die ursprüngliche reihenförmige Ortsanlage ist trotz der starken Verbauung zu erkennen

2) Das «Fischgrätenmuster» der Flur ist schon zu ahnen

3) Eine grobe Gliederung der Flur nach der Physiognomie fördert einheitlich wirkende Parzellenkomplexe zutage, die durch die Viehtriebwege zur Gemeindeweide, die Vys, begrenzt werden.

Der Vergleich mit der Flur vor der Flurbereinigung zeigt deutlich die Unterschiede.

1) Damals bestand ein zunächst verwirrendes «Fischgrätenmuster» schmaler und schmalster, durch Mäuerchen und Hecken voneinander abgegrenzter Parzellen.

2) Eine physiognomische Einteilung führt jedoch zu einer Gliederung der Flur, gleichzeitig zu einer Verfeinerung der vorhin gemachten Angaben: Ausgeprägte Strukturlinien begrenzen Parzellenkomplexe einheitlicher Natur.

3) Die Flur nördlich der Straße wird durch eine Linie begrenzt, die die streifig geregelten Parzellen in Ortsnähe von ungeregelten Block-Streifenverbänden weiter im $\mathrm{N}$ absetzen. Es handelt sich hierbei um eine alte Rodungsgrenze.

4) Der Besitz der Bauern konzentriert sich in bestimmten Räumen der Flur, abgesehen von dem südlich der Straße gelegenen Hof mit deutlichem Außenbesitz. Dies stützt ebenfalls die Annahme, daß eine einmalige Hufenflur vorliegt, darüber hinaus die Vermutung, daß der Ort ursprünglich teilweise einzeilig war.

Der nächstfrühere Plan (1870/1) ist nicht sehr vom Plan direkt vor der Flurbereinigung unterschieden. Interessant ist dagegen ein Plan von 1734, der sowohl den Zustand der Flur zu dieser Zeit als auch den von 1658/9 wiedergibt. Der Zustand von 1734 ist nach der Katasterkarte dieses Jahres mit schwarzer Tinte, der von 1658/9 nach den Besitzverzeichnissen dieses Jahres (den «reconnaissances») mit roter Tinte eingezeichnet. Der Nachteil dieses Planes beruht darauf, daß nicht alle Parzellen eingezeichnet sind, sondern meist nur die zinspflichtigen. 
La Brévine im Neuenburger Jura (links) und Lac de Taillères

(rechts). Man beachte die beidseits dem Talrand entlang

gereihten Einzelhöfe mit ihrer Waldhufenflur.

(Swissair-Photo AG, Zürich)




Bei der Untersuchung dieses Planes und der $\mathrm{Zu}-$ hilfenahme der Besitzverzeichnisse von 1658/9 ergibt sich, daß zwischen beiden Terminen eine Besitzzersplitterung stattgefunden hat. Einheitlicher hufenförmiger Besitz 1658/9 ist 1734 unter mehrere Eigentümer aufgeteilt, wie das Beispiel des Besitzes der Familie Lambelet zeigt.
Diese Tatsache entspricht der Bevölkerungszunahme nach dem 10jährigen Krieg. Dieser Vorgang zeigt sich auch darin, daß beispielsweise vier im Jahre 1734 selbständig aufgeführte Eigentümer Besitz haben, der auf einen einheitlichen 1658/9 zurückgeht:

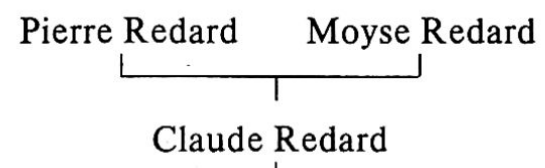

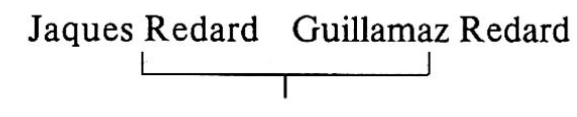

Nicolas Redard

Für die Zeit vor der Aufnahme dieses Planes existieren nur Besitzverzeichnisse, die sog. «reconnaissances» oder "extentes». Erhalten sind solche Besitzverzeichnisse aus den Jahren 1658/9 (von Marthy und Viret), 1558 (von Hory), 1429 (von Guyot de Lannoix) und 1340.

Insgesamt kann man sagen, daß die Angaben um so ungenauer sind, je älter die reconnaissance ist. 1658/9 kann man noch weite Bereiche an der Straße rekonstruieren, 1558 ist das auch noch teilweise der Fall. 1429 kann man nur mehr einige Felder lokalisieren, freilich wird die Reihenanlage der Parzellen auch durch diese reconnaissance bewiesen, dadurch, daß bei der Lagebezeichnung der einzelnen Parzellen immer der westliche und der östliche Anrainer genannt sind, das bedeutet, man hatte damals Streifenparzellen in hufenförmiger Anordnung. Außerdem nimmt der Grad der Parzellierung ab, je weiter man in die Vergangenheit zurückgeht.

Wesentlich für die Flurstruktur der Frühzeit von Les Verrières ist nun die extente von 1340, die älteste erhaltene, freilich nicht die älteste überhaupt. In dieser extente sind aufgeführt:

- Die Besitzungen der Haushaltsvorstände

- Die Grundstücke in ihrer Größe, manchmal ihrer Lage, manchmal mit 1 Anrainer und mit der zu zahlenden Abgabe

Die Rekonstruktion ist äußerst schwierig wegen der ungenauen Angaben. Ein Problem ist zunächst die Bedeutung mehrerer in der extente genannter $\mathrm{Be}$ griffe:
- massus (= mansus)

- posa (pose)

- falcata (faux)

Offensichtlich gibt es Unterschiede in der Bedeutungseinschätzung: die massi liegen immer in Ortsnähe und werden fast immer an erster Stelle aufgeführt. Das heißt, die massi waren hofnahe Felder, die falcatae und posae weiter entfernte Zusatzfelder. Massi, posae und falcatae sind Größenbemessungen. Die posae und falcatae sind größenmäßig eindeutig festgelegt:

Die posa ist die spätere pose neuchâteloise $=0,27$ ha Die falcata ist die spätere faux $\quad=0,54$ ha

Die Schwierigkeiten beginnen erst mit der Definition von massus. Massus oder mansus (im französischen maix oder mas) ist ja die lateinische Übersetzung von "Hufe» und hat wie dieser Begriff einen dauernden Bedeutungswandel zu verzeichnen gehabt. Einmal ist mansus eine Besitzparzelle mit daraufliegendem Hof (auch in der geographischen Bedeutung von «Hufe»), zum anderen eine Größenbemessung, und schließlich hat sich auch hier die Geographie dieses historischen Begriffes bemächtigt, so daß eine der besten Übersetzungen für «Hufendorf» "village en maix» (mansus) ist.

In unserer extente finden wir ganze, doppelte, halbe, viertel massi. Hier ist massus als Größenbemessung gebraucht. Ein massus hat 4-5 ha Flächeninhalt gehabt, das entspricht ungefähr 15 journaux (Tagewerken). 
Die Interpretation dieser extente ergibt:

1) Die Struktur der gereihten Streifen war 1340 vorhanden. Sie orientiert sich an dem vorher herausgestellten Grundgerüst der Flur.

2) Die streifig geregelte, in massi angegebene Flur reicht bis zu einer Rodungsgrenze.

3) Diese extente wurde aufgenommen, nachdem schon 3-4 Generationen in Les Verrières gelebt hatten. Seitdem ist wohl das Flurbild durch Realteilung etwas zersplittert worden.

4) $\mathrm{Daß}$ die ursprünglichen Hufen den physiognomischen Abschnitten entsprochen haben, ist möglich, kann aber nicht bewiesen werden. Eine Hufe hätte dann einen Flächeninhalt von 2-3 massi, also zwischen 10-15 ha gehabt.

Warum hat nun Les Verrières-de-Neuchâtel (und der benachbarte Ort Les Bayards) waldhufenartige Flurgliederung, andere Orte in derselben Situation jedoch nicht? Die physisch-geographischen Faktoren - das langgezogene Synklinaltal von Les Verrières - fallen kaum ins Gewicht. Diese hätten wegen des dauernden Wassermangels eher Haufendörfer oder Weiler um die wenigen Brunnen hervorgebracht.

Es handelt sich hier vielmehr um eine grund- oder territorialherrschaftliche Einflußnahme mit dem Ziel, zum eigenen Nutzen (Steuern und Abgaben) Siedler anzusetzen.

Dafür spricht eine rechtliche Sonderstellung der Bewohner von Les Verrières, dessen Gründungszeit an die Wende vom 12. zum 13. Jh. zu setzen ist. Die erste erhaltene Befreiungsurkunde, die sog. "charte d'affranchissement» stammt bereits von 1337 , ist also ausgesprochen früh. Hierdurch wurden die Bewohner von Les Verrières von der Main morte befreit, die in etwa dem deutschen Heimfallrecht entspricht und besagte, daß nach dem Tod eines Untertanen dessen Besitz an den Herrn zurückfällt.

Das war ein weitgehendes Privileg, das die Bewohner des früher besiedelten benachbarten Val-de-Travers erst Jahrhunderte später erhielten. Diese Befreiungsurkunde war jedoch sicher nicht die erste. Das wird bewiesen durch die Tatsache, daß während des 16 . Jahrhunderts im Zuge von Streitigkeiten mit Nachbargemeinden eine Untersuchung über den rechtlichen und sozialen Status der Bewohner von Les Verrières hergestellt wurde. Dabei ergab sich, daß sie «franc-habergés» (freie Siedler) waren, die bei der Kolonisation mit Land versehen wurden («ammassez», von massus!).

Worin sich diese Besserstellung genau ausdrückte, ist nicht mehr faßbar. Wesentlich erscheint jedenfalls, daß Les Verrières einer planenden Einflußnahme des Territorialherrn, des Grafen von Neuchâtel, seine Entstehung verdankt.

Les Verrières-de-Neuchâtel wurde als Beispiel für die Waldhufendörfer des Jura geschildert. Ihnen allen - mit einer Ausnahme - ist es gemeinsam, $\mathrm{da}$ sie ihre Entstehung einer planvollen Einflußnahme von Grundherren im Verlauf des 12.-14. Jh., der ersten Rodungsperiode des Jura, verdanken.

\section{Das Tal von La Brévine}

Das Tal von La Brévine ist ebenso wie das benachbarte Tal von Les Ponts-de-Martel ein charakteristisches Synklinaltal des hohen Faltenjura. Durch das Moor, das den gesamten Talgrund in seiner ganzen Länge einnimmt, waren bereits die Leitlinien der Besiedlung gegeben: Es kam zur Herausbildung von zwei Siedlungsreihen zu beiden Seiten des Moors. Die heutige Siedlungs- und Flurstruktur ist durch gereihte Einzelhöfe und hofanschließende Breitstreifeneinödparzellen gekennzeichnet.

Teilweise existieren zweite und dritte Häuserreihen oberhalb der Talsiedlungen. Diese sind aus später verselbständigten Maiensässen und Alpweiden, die sich ursprünglich auf derselben Hufe befanden wie die Talgüter, hervorgegangen. Die Länge der Hufen erreicht heute noch annähernd $3 \mathrm{~km}$, die Fläche der ehemaligen Hufen hat bis zu 200 ha betragen.

Interessant ist, daß das Besitzgefüge senkrecht zum talparallelen Verlauf des Nutzungsgefüges angeordnet ist. Anzumerken bleibt schließlich, daß die Konzentration von kommunalen Einrichtungen und die Anlage kleinerer Industriebetriebe zur Herausbildung von baulichen Kernen innerhalb dieses Einzelhofsiedlungsgebietes geführt hat. In unserem Fall sind das die Orte La Brévine und La Chaux-duMilieu.

Pläne aus dem 18. Jh. und Besitzverzeichnisse aus früherer Zeit zeigen dieselbe hufenförmige Struktur. In den reconnaissances der Mitte des 16. Jh., den ältesten erhaltenen, sind zusammenhängende $\mathrm{Hu}$ - 
fen aufgeführt, die der vermuteten Größenordnung nahekommen. Die Besitzungen sind weitaus größer als die im Tal von Les Verrières.

Die gereihten Einzelhöfe mit Waldhufenflur entstammen einer späteren Ausbauphase des Jura (16./ 17. Jh.). Ihre Anlage verdanken sie darüber hinaus nicht grundherrschaftlicher Initiative. Das ersieht man daraus, daß sie in Gebieten verschiedener Herrschaften, aber immer in Bereichen gleicher natürlicher Ausstattung vorkommen.

Gesiedelt wurde hier hauptsächlich von Kolonistengruppen (so aus Le Locle), denen, nachdem sie gesiedelt und die Grundherrschaften vor vollendete Tatsachen gestellt hatten, Sonderrechte zugestanden wurden, um sie am Abwandern zu hindern. Die regelmäßige Siedlungs- und Fluranlage geht also nicht auf grundherrschaftliche Initiativen, sondern auf eigene Absprachen der Siedler untereinander zurück. Das ist der Hauptunterschied zur Genese der Waldhufendörfer.

\section{3. Übertragung des Waldhufenprinzips aus anderen Gebieten}

Bei den Waldhufensiedlungen des Jura konnte keine Übertragung des Systems der gereihten Streifen aus anderen Gebieten festgestellt werden. Es handelt sich hier wohl um eine unabhängige Entwicklung. Die Entfernung zu den nächstgelegenen Waldhufensiedlungen ist wohl auch für eine Übertragung zu groß (Odenwald, mittlerer Schwarzwald).

Es soll freilich nicht bestritten werden, daß solche Übertragungen stattgefunden haben, es gibt jedoch genügend Beweise dafür, daß Hufensiedlungen auch unabhängig voneinander entstehen können. Die Hufenflurgestaltung schien und scheint eben zweckmäßig und dem Gelände gut angepaßt. Bei einem gewissen $\mathrm{Ma} \beta$ an Planung oder Absprachen der Siedler untereinander können Hufensiedlungen eben nicht das Ergebnis äußerer Beeinflussung, sondern dasjenige eigenständiger Entwicklung sein, und so war es m. E. auch im Jura, bei den beiden physiognomisch und genetisch unterschiedlichen Typen, dem Waldhufendorf und der Einzelhofreihe mit Waldhufenflur.

\section{Literaturauswahl:}

BARTZ,F.: Französische Einflüsse im Bild der Kulturlandschaft Nordamerikas; in: Erdkunde, 1955, S. 286 bis 305

BLOCH, M.: La société du haut moyen âge et ses origines, in: Journal des savants, 1936, S. 403-420

BLOCH, M.: Les caractères originaux de l'histoire rurale française, $1931\left({ }^{2} 1952\right)$

BLOHM, R.: Die Hagenhufendörfer in SchaumburgLippe; in: Schriften des Niedersächsischen Heimatb., Neue Folge, 10, Oldenburg, 1943

CONRAD, H.: Deutsche Reichsgeschichte, 2. Auflage, Bd. I, Karlsruhe, 1962

DAVEAU, S.: Les régions frontalières de la montagne jurassienne. Etude de géographie humaine. Mémoires et documents de l'institut des Etudes Rhodaniennes, Université de Lyon, Nr. 14, 1959

DEMANGeon, A.: La France, 2. Teil, Band 6 der Géographie Universelle, 1946

DION, R.: Perspectives de recherches sur l'évolution des types d'habitat rural en France, in Comptes Rendus au congrès international de géographie d'Amsterdam, 1938, Travaux des sections A-F, S. 62-72

GRADMANN,R.: Das ländliche Siedlungswesen des Königreiches Württemberg, Forsch. z. dt. Landesund Volkskunde, Band 21, 1913

GUTERSOHN, H.: Geographie der Schweiz, Band 1: Der Jura, 1958

HABBE, K.-A.: Das Flurbild des Hofsiedlungsgebietes im mittleren Schwarzwald am Ende des 18. Jahrhunderts. Diss. rer. nat. Freiburg, 1957, in: Forsch. z. dt. Landeskunde, Band 118

HABBE, K.-A.: Die «Waldhufensiedlungen» in den Gebirgen Südwestdeutschlands als Problem der systematischen Siedlungsgeographie, Ber. z. dt. Landeskunde, Band 37, Heft 1, 1967, S. 40-52

JUILLARD,E. und MEYNIER: Die Agrarlandschaft in Frankreich. Forschungsergebnisse, in: Münchner geogr. Hefte, Nr. 9, 1955, übersetzt von w. HARTKE KÖTZSCHKE, R.: Hufe und Hufenordnung in mitteldeutschen Fluranlagen; in: Wirtschaft und Kultur, Festschrift zum 70. Geburtstag von Alfons Dopsch, Baden, Wien, Leipzig, 1938, S. 243-265 
KÖTZSCHKE, R.: Ländliche Siedlung und Agrarwesen in Sachsen; in: Forsch. z. dt. Landeskunde, Band 77, 1953

KREISEL, W.: Structures agraires de "Waldhufendorf» dans le Jura; in: Revue de Géographie de Lyon, Band 44, 1969, no. 1, S. 85-113

KREISEL, W. und SCHOOP, W.: Landnahme und Kolonisation im französischen und schweizerischen Jura und im nordöstlichen Andenabfall Boliviens. - Eine vergleichende Untersuchung zur Besiedlung zweier bewaldeter Gebirgsregionen, in: Geographica Helvetica, Heft 4, 26. Jg., 1971, S. 181-186

KREISEL, W.: Siedlungsgeographische Untersuchungen zur Genese der Waldhufensiedlungen im Schweizer und Französischen Jura mit einem Ausblick auf die bevölkerungs- und agrargeographische Entwicklung, Aachener Geographische Arbeiten, Heft 5, 1972

KRENZLIN,A.: Probleme nordostdeutscher und ostmitteldeutscher Flurformenforschung; in: Archiv f. Landeskunde $u$. Volksforschung, Band 4, 1940

KRENZLIN, A.: Historische und wirtschaftliche Züge im Siedlungsformenbild des westlichen Ostdeutschland; Frankfurt Geogr. Hefte, 27-29. Jg., Einz. H., 1955

KRÜGER,R.: Typologie des Waldhufendorfes nach Einzelformen und deren Verbreitungsmustern, Gött. Geogr. Abh., Heft 42, 1967

LEBEAU, R.: Carte des Formes d'Habitat Rural de la Chaîne Jurassienne, Suisse et Française; in: Regio Basil., Hefte für jurassische und oberrhein. Landeskunde, Cahiers de Géographie jurassienne et rhénane, II 1, 1960, S. 19-34

LEBEAU, R.: Les Grands Types des Structures Agraires dans le Monde; in: Initiation aux Etudes de Géographie, 1969

LOEW,F.: Les Verrières, la vie rurale d'une communauté du haut-Jura au moyen-âge, 1954

LÜTGE,F.: Dt. Sozial- und Wirtschaftsgeschichte, Berlin-Göttingen-Heidelberg, 1952

LÜTGE,F.: Geschichte der dt. Agrarverfassung vom frühen Mittelalter bis zum 19. Jh., Dt. Agrargesch. 3, Stuttgart, 1963

MANSHARD, w.: Die geographischen Grundlagen der Wirtschaft Ghanas. Unter besonderer Berücksichti- gung der agrarischen Entwicklung. Wiesbaden, 1961, 308 S. Kölner Geogr. Arb., Sonderfolge Beiträge z. Landeskunde Afrikas, Heft 1

MARTIN,R.: Die ländliche Siedlungsgestaltung im Schwarzwald; in: Zeitschrift Gesch. d. Oberrheins, Neue Folge, Band 45, 1932

MONTANDON,L.: L'établissement des Loclois dans la vallée de La Brévine au $16^{\mathrm{e}}$ siècle, in: Musée Neuchârelois, nouvelle série, Jgg. 37, 1950, S. 41-58

NITZ, H.-J.: Die ländlichen Siedlungsformen des Odenwaldes; in: Heidelberger Geogr. Schriften, Heft 7, 1962

NITZ, H.-J.: Das Alter der Langstreifenfluren, Ber. zur dt. Landeskunde, Bd. 29, 1962

RICHTËR, W.: Historische Entwicklung und junger Wandel der Agrarlandschaft Israels, dargestellt insbesondere am Beispiel Nordgaliläas; Kölner Geogr. Arb., Heft 21, 1969

ROHMER, M.: Les structures du parcellaire rural française, origines et évolution, in: Géomètre, Juni 1969, 6, S. 31-51

SCHOOP, w.: Vergleichende Untersuchungen zur Agrarkolonisation der Hochlandindianer am Andenabfall und im Tiefland Ostboliviens; Aachener Geogr. Arb., Heft 4, 1970

SCHWARZ, G.: Allgemeine Siedlungsgeographie, Berlin ${ }^{3} 1966$

SCHWIND, M.: Der japanische Bauer, seine Arbeit und sein Dorf; in: Japan, von Deutschen gesehen, hrsg. von M. Schwind, 1943, S. 113-119

SCHWIND, M.: Der japanische Bauer, seine Arbeit und sein Dorf; in: Japan von Deutschen gesehen, 1954-55, S. 155-167

UHLIG, H. (Hrsg.); LIENAU,C. (Red.): Flur- und Flurformen, Materialien zur Terminologie der Agrarlandschaft, Bd. I, Gießen, 1967

WAIBEL,Z.: Die europäische Kolonisation S-Brasiliens, bearbeitet und mit einem Vorwort versehen von G. Pfeiffer, Coll. Geogr. 4, Bonn, 1955

zSCHOCKE, H.: Die Waldhufensiedlungen am dt. Niederrhein. Kölner Geogr. Arb., H. 16, 1963

ZSCHOCKE,H.: Rezension von R. Krüger, Typologie des Waldhufendorfes nach Einzelformen und deren Verbreitungsmustern, in: Geogr. Zeitschrift, Heft 1, März 1970, S. 57 f. 